\title{
Apparatuses \& Constructed Narratives: The Imaginary Life Of Cappadocia
}

\author{
CARLA ARAMOUNY \& SANDRA RISHANI \\ American University of Beirut
}

This paper presents the work done during a Vertical Design studio, held at the Department of Architecture and Design in Beirut, and discusses the studio design methods that evoke experimental model making and narrative programming. The work presented develops on themes of locality, landscapes as systems of reference for design, physical constructs as inherent design machines, and fictional narratives as programming devices. Through the use of complex drawings and dynamic models, the studio intervened on the region of Cappadocia in Turkey, with its complex land formations, proposing new visions for a unique site where architecture and landscape coalesce.

\section{INTRODUCTION}

In "The Fairies' Pits and Towers", an undergraduate travelling design studio undertaken at the American University of Beirut, Department of Architecture and Design in Spring 2015, the 'natural' land formation of the region of Cappadocia, Turkey, with its complex matrices of subterranean dwellings, were the initial inspiration. 24 Students from both $3 \mathrm{rd}$ and 4 th year levels of the Architecture undergraduate program participated in the studio. Our interest was to tackle the complex topological landscapes, from both, a morphological/ historical understanding and a design perspective inspired by fiction. The studio involved three levels of design inquiry: local landscape, spatial constructs and user narratives, around which the projects evolved in active explorations, moving between dynamic time/space relations to physical and spatial interventions. This paper will detail and present the work done throughout the semester from the conceptual and analytical standpoints to the design application.

\section{THE LANDSCAPES OF CAPPADOCIA}

Cappadocia, a region in Central Anatolia known for its unique landscapes of soft rock formations or "fairy chimneys", was created through processes of erosion and volcanic deposition forming a vast landscape of hills and valleys. These geo-morphological conditions have long dictated the spatial organization and cultural development of these historical landscapes, where locals inhabited their land by burrowing and forming continuous chains of underground dwellings. The quality of the rock, soft ash in the center with a solid basalt layer on top, created both a soft yet structurally consistent forming material that allowed inhabitants to modulate intricate and distinctive dwellings throughout history. Constant prosecution in the zone's history led to a proliferation of these complex subterranean constructs, extending to vast distances and depths. The locals continued to inhabit these chains of caves well into the 20th century, until the 1960s, when the Turkish government started relocation efforts, and moved the inhabitants from dwellings under risk to neighboring villages. Many of these fairy chimney constructs and underground cities were vacated to become museums and touristic sites. The recent status of Cappadocia, and more specifically the Goreme open-air museum as an UNESCO World Heritage site, enforced another shaping influence, one that favors tourism over culture, and promotes landscape as a commodity. Through the promotion of their unique landscape, the different towns in the hilly region offer their visitors new spatial and touristic experiences. This status has subsequently pushed many more inhabitants to move out from the very popular touristic towns to nearby areas, leading to a loss of local traditions and cultures and their slow replacement by souvenir replicas and hotels chains.

These conditions formed our initial interest in the site as an area where landscape, culture and tourism strongly overlap. The interest in Cappadocia's landscape as the starting point was triggered by the scale and uniqueness of its formations and its distinctive integration of spaces and land. As students and faculty coming from nearby Beirut, we were interested in exploring an unfamiliar territory as a way to reflect on our own landscapes and the relationship they inflict on local culture. In contrast to the similarity regionally and internationally of contemporary design forms and constructions, landscape is considered here as a truly "local" condition, one that allows contextual understanding of spaces and people. The extended landscapes of volcanic formations accordingly became the starting reference for explorations and architectural interventions

\section{STUDIO METHODOLOGY}

The framework within which the studio operated involved starting with an investigation of existing accessible data and popular online culture 


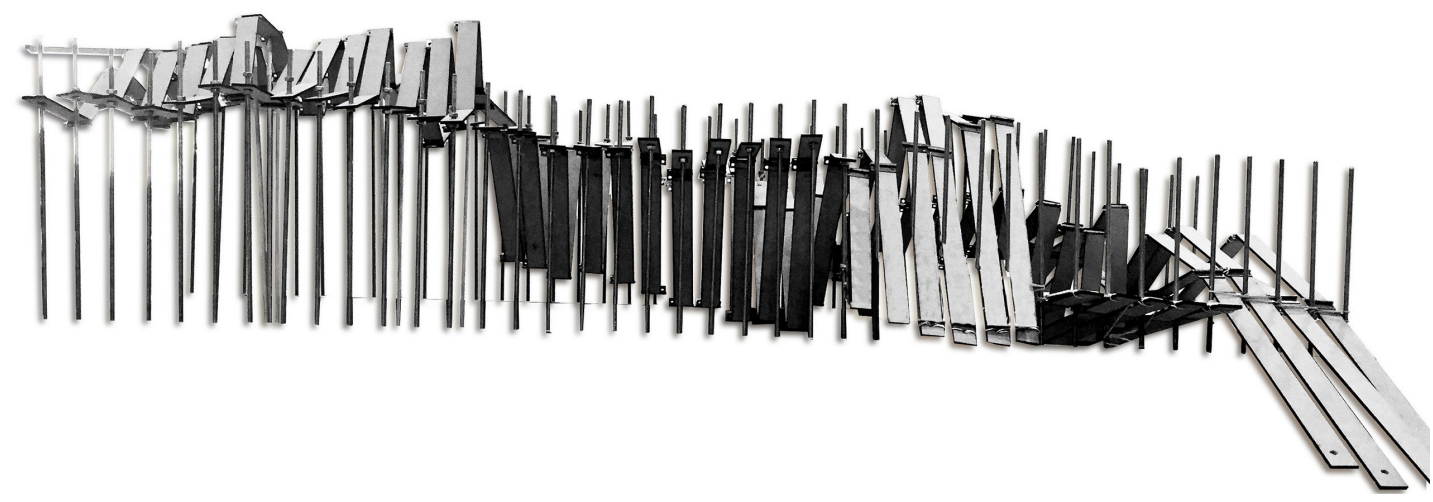

Figure 1: Ghida Chehab and Sarah Abiad - White Valley apparatus in ribs of wood with steel connections

prior to going to the site. The intention was to formulate a personalized assumption of that landscape and its inhabitants, basing it first on its touristic image and the dynamic aspects of its landscape. After the initial reconstruction of the landscape, the idea was to visit Cappadocia towards the middle of the semester, and to reconcile the students' assumptions and proposals with the reality of this landscape and its tangible characteristics. With that in mind, the students were asked to investigate this new environment remotely yet be always conscious of their own external inputs and preconceived ideas as mechanisms that affect their design interventions. Within this main framework, and before the site visit, the studio design methodology was developed around three main phases that moved from analytical understanding to conceptual devices and proposals. The three phases were as follow: landscape analysis, apparatus/spatial constructs, and fictional narratives/ interventions.

\section{LOCAL LANDSCAPE ANALYSIS}

The first phase involved investigating landscape as a system of reference by partaking a detailed analysis of the different shaping processes that have occurred in time, whether geological, environmental, social, historical, or cultural and to use such processes to create visual and physical reconstructions of the site. As the students have never been to the area, they worked to generate an understanding of the zone based on readily accessible data that sometimes tends to be touristic and slightly stereotyped. Through available online maps, Google earth imagery, tourist recounts and landscape videos, they started their investigations and mappings. However, the students were expected to look at those data in an alternative manner. Rather than seek the immediate and the generic, they were asked to dig deep for unforeseen conditions, specificities, and dynamic relationships that change through time. Conditions such as geologic movements, erosion, water cycles, social and economic flows, were all tackled and researched in depth. The students were divided into groups of two, each of which focused on a specific theme that became their lens of inquiry for the site.

The students' analysis took on several recurring approaches. Some students focused their research on a geomorphological analysis, attempting to describe the relationships between the land formations and their shaping processes, and thus focusing on geography and land tectonics as dynamic systems. One of the groups tackled the formations of the White valley in Cappadocia', located between the towns of Uchisar and Cavusin. In this case tourist images of the valley online invited this group to delve into understanding the conditions that spatially created the white valley. The relationship of wind and water in sculpting the valleys was mapped with their varying intensities and directionality. The tectonic plate movement with the changing rates of erosion was meticulously analyzed to recreate a narrative of the valley's evolution through time. Through mappings and explorative models, the students re-formulated their site as an active system of forces and resulting morphologies.

A second group ${ }^{2}$ remapped the absurdities of the landscape with its fairy chimney formations. The fairy chimney typologies, composition, structural integrity as well as their modes of formation in time became their main focus. They assimilated the fairy chimney characteristics to four main factors, which affect their shape, life span and strengths, including slope, climate, geologic layering and their qualities. They showed that the fairy chimneys form in different assemblies, whether joined together, grouped in sub-formations, or differentiated individually as conical structures with thick basalt capping. The students formulated their physical re-construction of the landscape as a density of points by allocating to the fairy chimneys various intensities and typologies.

Other students' research ${ }^{3}$ tackled more ephemeral relationships between the tourist industry and its implication on land and sky. The hot air balloons' continuous movements, frequency, trajectory and their density were mapped through time-lapse videos, and were overlaid with the density of fairy chimneys below. The navigation of these balloons was shown to rely heavily on wind and ground conditions. The combined trajectories of all the balloons were traced onto various diagrams that revealed the moments of watching, interacting, intervening and disturbing the landscape.

\section{SPATIAL CONSTRUCTS /APPARATUS}

In the second phase, following their extensive site research, mappings and analysis, the students moved to produce physical models that represented the flow of these data in time. They were asked to follow on their select lens of inquiry and to translate their research three dimensionally into a physical construct/ or apparatus. These conceptual yet analytical apparatuses were considered as active design machines, 

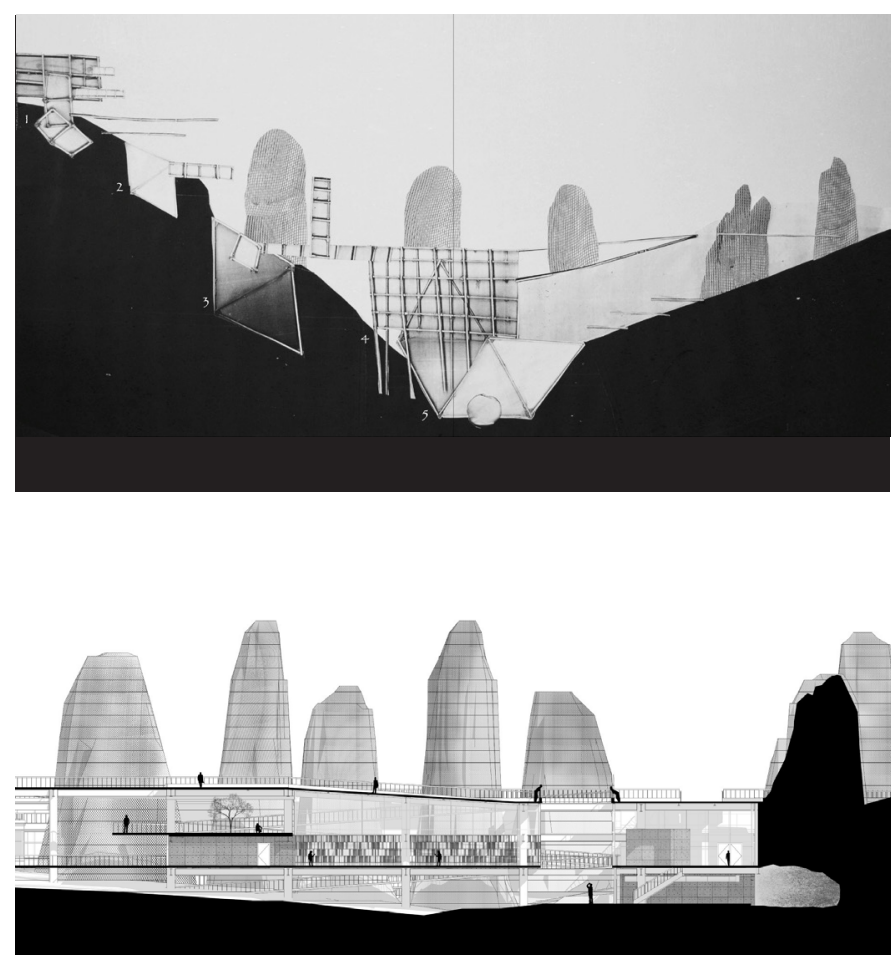

Figure 2: Mohamad Deeb and Tina Najia - "Land of the Uncanny" Narrative composition and sectional intervention

with embedded spatial and organizational logic. They were envisaged as the basis of the idea, a "body without organs"4 that organized, programmed and imagined a new fiction for the landscape. The studio heavily encouraged experimentation and the use of hands-on model making and drawing. The models expressed spatial and formal relationships in time, and deployed actions, activities, moments, and varied uses. The previous figure [Fig. 1] shows the project apparatus depicting the forces of the White valley ${ }^{1}$, where wind and water actions are used to form two types of movements and joints. Length and amount of connections in the wood and steel members reformed the valley into a continuous spatial skeleton that the students could control and manipulate to redesign the space of their model in various typologies. This new landscape represented their own interpretation of the forces at play, and a site for a possible new intervention.

From the previous research described on hot air balloon trajectories ${ }^{3}$, the physical apparatus followed the initial mappings and was conceived as a translucent textile that formed the space between the varying trajectories and positions of the balloon flights and the fairy chimney formations. The fabric was reformed and manipulated continuously by the pulling of the strings in the positive $y$ and $z$ direction. The interest of the students was to understand the interconnectedness of the balloons and the fairy chimney formations, and to reveal through the fabric the intermediate space that both the tourists and the unique formations generate.

In another apparatus ${ }^{5}$, the deep Underground cities of Cappadocia, mainly in the town of Derinkuyu, were tackled as a system of intricate spaces and inflations, relying on above ground pumping of air, and adjacencies of water pockets to reform and redesign voids. The underground city analysis revealed a strong interrelation between above ground dynamics and underground spatial typologies. Within the eight stories deep excavated tunnels and chambers, were long dug vertical wells that relay to the underground air and water channels. The model as such was depicted as a series of syringes and balloons, hung on a wood and thread matrix, connected to a lower space, relieving and elevating various inflations and deflations.

\section{USER NARRATIVES}

Apparatuses became complex machines devoid of location and instead localized relationships that exist in the standalone object. Students were then asked to move to the third phase and understand the apparatuses they designed as relationships with human interaction, by injecting in them a fictional proposal, a user narrative involving both locals and tourists. Their narratives, conceived as programming devices, were visualized as complex collages of lines, drawings and texts. Drawing, again like the physical constructs, was conveyed as an active design tool, rather than a mere representation. Through the narrative, the aim was not to design for a fixed typology of program, be it a house, a hotel, or other, but rather to reimagine the components of that typology through involving new possibilities and activities that could take place. Design was thus looked at as a process of reinvention where "invisible" components of any given program were rearticulated and reimagined through drawing.

“... Design has an invisible component, namely an organizational ... dimension over which the designer always exercises a certain influence yet which, given the way we classify our environment in terms of objects, tends to remain hidden." 6

For example, rather than designing a "house for a visitor" based on already existent typologies, the students would explore the activities, actions, and events that revolve around the story of "inhabiting a space as a visitor in Cappadocia," and turn these events into spaces that connect to the local landscape. This landscape, an inherent part of all the students' proposals, was not seen as external to their projects but rather as part of the same extension, where all "activities" connect between the external and the internal spaces. [Fig.2]

Each group had to form their various spatial and user proposals on a single A0 sheet. The drawing was intended to convey a holistic spatial composition, combining sectional drawings with perspectival projections. Each drawing became representative of the students' personalized depictions of these newly formulated landscape. To start the reconciliation between their preconceived depictions and reality, students had to exchange drawings in a four-hour session. They had to work over another group's drawing, helping in clarifying and visualizing inherent spatial conditions. Authorship, or the lack of authorship, became a critical issue in this exercise providing students with the leeway to look at their drawings as self-organized objects or manifestations, and allowing them to further influence their proposals after the actual visit to the site.

The student narrative ${ }^{5}$ on the Underground city of Derinkuyu utilized photocopied, scaled, and collaged fragments of drawings in mixed media, combined into a single composition. The drawing/narrative was used 


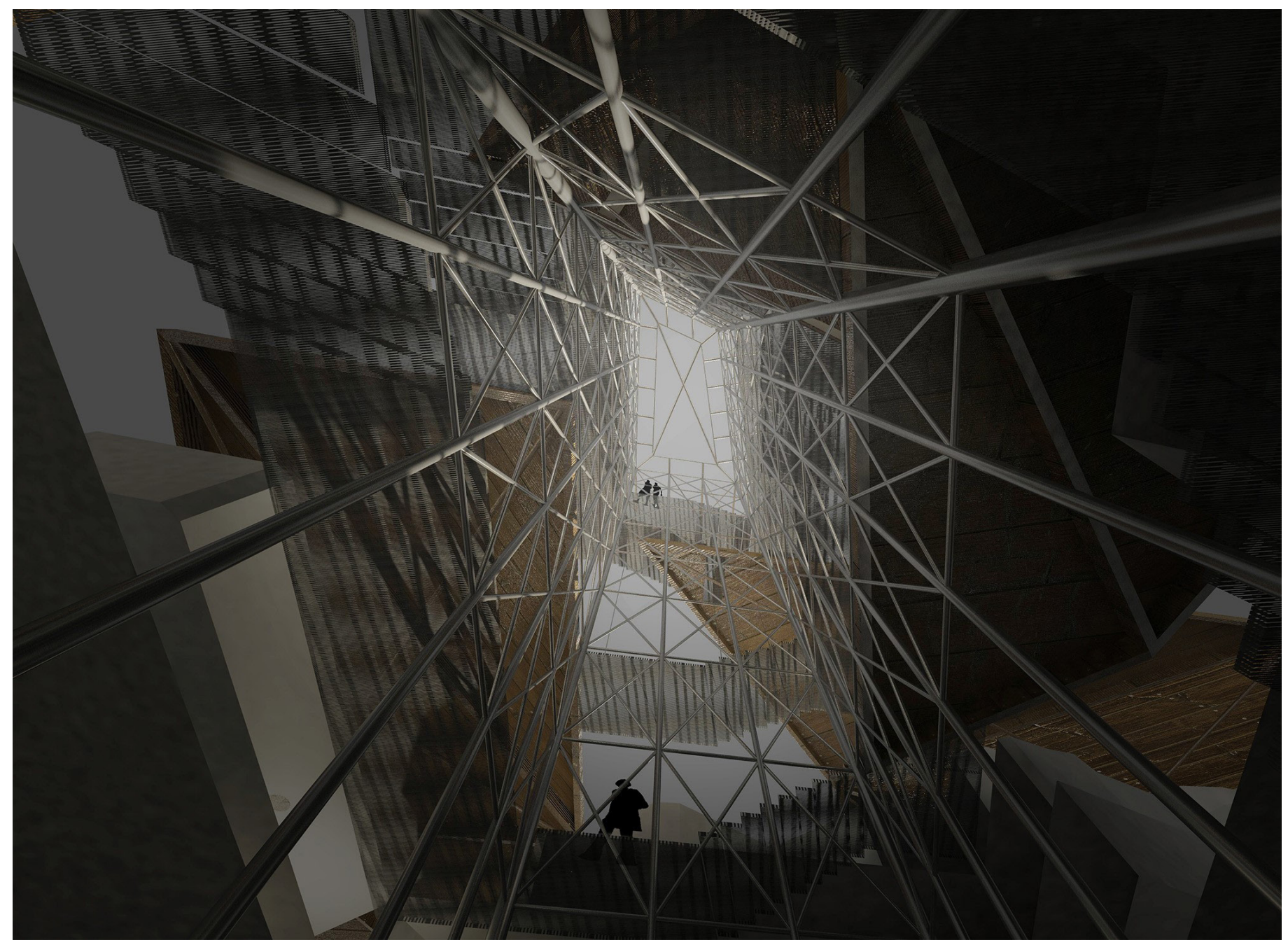

Figure 3: Ismail Hutet and Mariam Yassin - "Adaptive Landform" internal tower projected from landscape above to the underground

to depict a complex web of "underground" circular spaces and vertical shafts, with activities tailored to tourists, and town residents, including a brothel, a circus, and a fight club. Entrances for the various visitors, their service quarters, and the space of interaction among the different users, became the prime interventions on the paper. The underground was conceived thus as the "other", the unimagined covert place and program that lie deep beneath, catering to the influx of tourism.

\section{AFTER CAPPADOCIA}

This initial process was all done without visiting the region of Cappadocia, with the intent of remotely constructing an imagined or alternate understanding of the site. After the construction of the narratives, the studio travelled to Cappadocia, to reconcile the imagined with the tangible. With Cappadocia's visit came realizations of the actual scale of these formations, their sheer magnitude yet also their mundaneness, the reality of the locals and tourism, and their mutual commodification of the landscape. From that point on, the students actively re-articulated their proposals, inputting newfound understandings and on-site observations. Their proposals evolved into localized spatial interventions, with the intent of forming locally bound architecture that mediate between the topological landscape, the local culture, and the influx of tourism. [Fig.3] The various proposals became thus much more articulate, dealing with notions of scale and local understanding. The students started zooming into specific select areas to form their interventions and narrow down their scale. Their previous imaginary narratives were pushed to reconcile actual conditions of site, ground and user groups. In Cappadocia, they talked with the local inhabitants, trying to understand their culture, connection to the landscape, and lives in the remote region.

Inspired by a meeting with a local activist on the preservation of local cultural heritage, and based on their previous mappings of flows of tourists and locals, one student project ${ }^{7}$ moved to propose near the town of Goreme a multi- educational facility catering to locals and tourists. The fairy chimneys' real scale became a guiding factor for the design of the project as a floating mega-structure maneuvering in between and selectively interjecting in the landscape. The program involved an observatory, a public educational facility and tourist activities of performance spaces. [Fig. 2]

The balloon research project ${ }^{3}$ reconciled the balloon trajectories with the fairy chimney landscape by proposing platforms for take off and tourist vertical connections to the already existing carved spaces of 


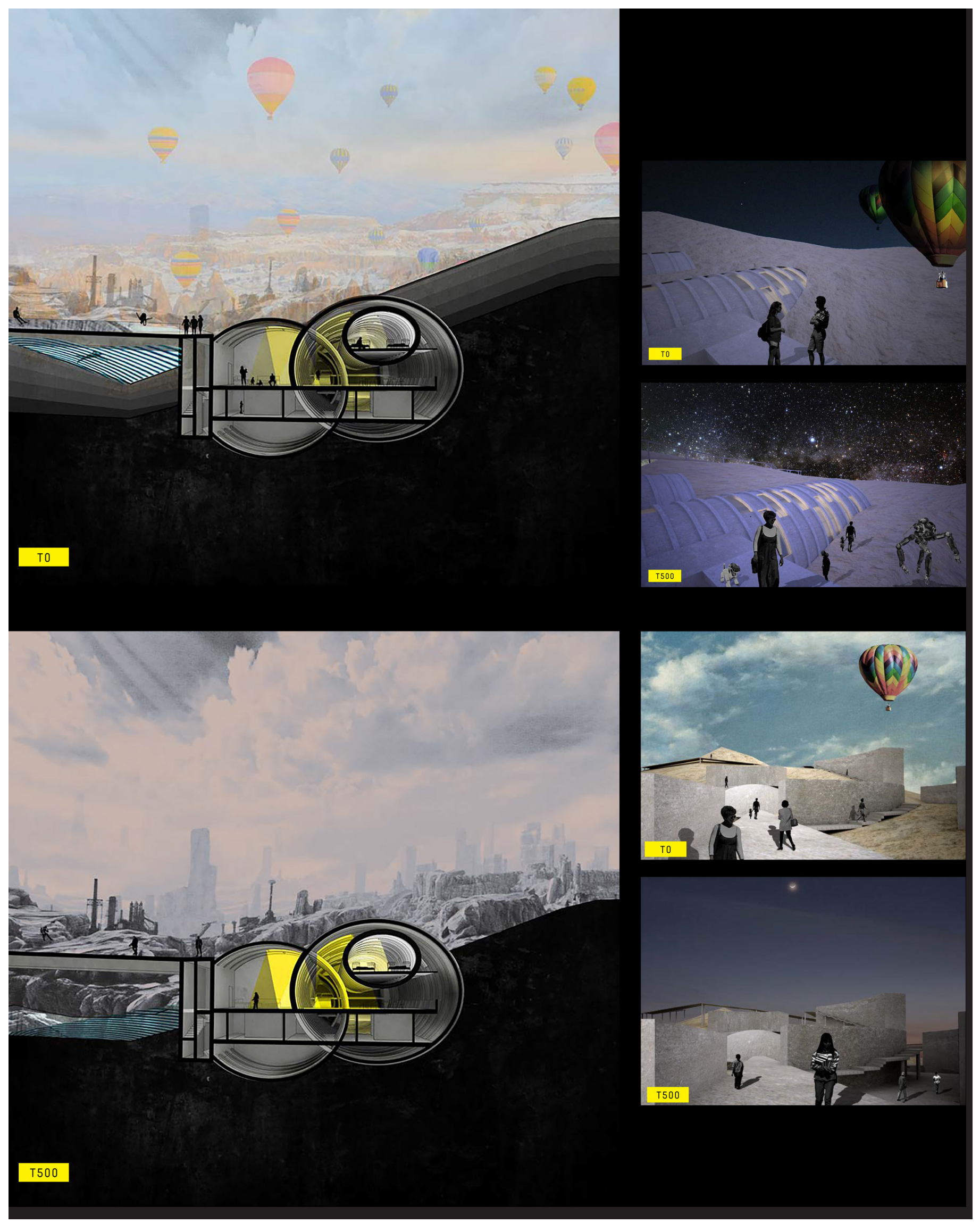

Figure 4: Alia Bader and Lori Kharpoutlian - "Anthropo-Tectonics" scenarios of the project at time frames T- 0 and T-500 years 
the chimneys. The students' personal experience of the site and its fairy chimneys made their intervention much more meticulous and less imposing. As these formations are considered heritage, local code forbids carving more spaces in them, but allows using their spaces in the state they are in. The students thus attempted to link internally to the already existing spaces within the group of fairy chimneys. They integrated their program with horizontal connections to these spaces, allowing tourists to move freely in and around them as they ascend towards the balloon lift-off decks. Their position as they move up in the landscape is reminiscent of the hot air balloon experience hovering above.

\section{ARTICULATED INTERVENTION}

One of the exemplary projects undertaken in this studio was one that looked at the site not as a static place but rather as a continuously changing landscape in time, where processes of erosion, land loss and water run-off produce through decades new topographic manifestation. The students' final proposal ${ }^{8}$ for the area of Avanos was described as an "anthropo-tectonic tool." Their research started on the influx of refugees to Turkey, particularly with the Syrian conflict, and the areas and shelters that they hid in. The landscape of Avanos, as in all Cappadocia, was modulated over time, through natural forces and its people's interventions, allowing for below ground shelters and concealed settlements. Factors of erosion in the area's history had slowly revealed and concealed further different sections of the intricate inner constructions.

Inspired by these transformations and attempting to understand their intensity over time, the students meticulously surveyed coordinates and altitudes, comparing slope to erosion intensity. They further linked erosion to wind exposure, orientation, and water run-off speed, and added to them factors such as land use and soil/rock type, to calculate the erosion rates of every patch of land. The final matrix conveyed in mappings and physical models became their site. The resulting apparatus allowed them to visualize the physical site and its future changes spatially and topographically. The site was thus looked at as a collection of future possible pits and peaks.

Their user narrative proposed an interactive fiction between tourists and refugees, and was constructed in several moments on a datum that evolves in time. Their fictional proposal detailed a project where refugees and tourist can inhabit two co-existing spaces, one below ground and the other above ground, completely unaware of one another. The geological layer that covers these spaces, for shelter and protection, was imagined to gradually peel away to turn them into exposed spaces for exploration. The refugees' spaces, designed to be concealed underground, will slowly remerge in five time frames, extending up to 2000 years, turning gradually into land ruins. As the land layers erode, the tourist program of pathways and visitors' center hovering above would slowly disintegrate as the two structures begin to merge, and their relationship to one another evolves.

The proposal thus moved away from the conventional perception of site as a still context in which a building sits, and viewed it instead as an evolving receptacle for the project. The project was designed in sections and models, with each section depicting five time frames of future centuries, and each ground layer representing the eroded landscape's position on that consequent time frame [Fig.4]. As described by the students, "the project is not one intended to be seen at one point in time, but rather over a course of thousands of years." It can be seen as an experimental study of the dynamics of a site, and the resulting change in the way a building can be perceived. It considers architecture as a continuous evolving repository between land, program, user groups and their interrelations.

\section{CONCLUSION}

In summary, the studio attempted at engaging landscape systems through an experimental design method, relying heavily on representation as a design machine, and framing it within a dialectical relationship between local understanding and personal narratives. It was an experimental quest for architecture students to discover new terrains and inject in them narratives of possible fiction. Through remotely addressing the select area's landscape and culture, the studio involved design interventions that mediate between the designer's personalized impressions and analysis, and the reality of the land under consideration. In that, the approach allowed students the chance to experiment with various potentials, from spatial typologies to fictional devices. The result was a multitude of projects sited in the undulating smooth landscape, integrating in moments, and in others imposing a new reading for an imagined Cappadocia.

\section{ENDNOTES}

1. Project by students Sarah Abiad and Ghida Chehab

2. Project by students Romy Sayah and Cynthia Atoui

3. Project by students Faissal Yatim and Yasmine Arkadan

4. "body without organs" notion by philosopher Gilles Deleuze, depicting the underlying forces and formalizing schemes inherent in the make-up of a "body"

5. Project by students Betina Abi Habib and Andrea Comair

6. Quote taken from Lucius Burckhardt, "Design Is Invisible" (1980)

Project by students Mohamad Deeb and Tina Najia

8. Project by students Alia Bader and Lori Kharpoutlian, winner of the Dean's Award for Creative Achievement (2015), FEA/AUB 